\title{
Efficacy of Fulvestrant in Women with Hormone- Resistant Metastatic Breast Cancer (mBC): A Canadian Province Experience
}

\section{Samitha Andrahennadi}

University of Saskatchewan College of Medicine

Amer Sami

Saskatoon Cancer Centre

Kamal Haider

Saskatoon Cancer Centre

Haji Chalchal

Allan Blair Cancer Centre

Duc Le

Saskatchewan Cancer Agency

Osama Ahmed

Saskatoon Cancer Centre

Mita Manna

Saskatchewan Cancer Agency

Ali El-Gayed

Saskatchewan Cancer Agency

Philip Wright

Saskatoon Cancer Centre

Shahid Ahmed ( $\nabla$ shahid.ahmed@saskcancer.ca )

University of Saskatchewan https://orcid.org/0000-0002-2099-698X

\section{Research Article}

Keywords: Hormone receptor positive breast cancer, fulvestrant, survival, hormone resistant, visceral metastases

Posted Date: May 24th, 2021

DOl: https://doi.org/10.21203/rs.3.rs-538054/v1

License: (c) (1) This work is licensed under a Creative Commons Attribution 4.0 International License. Read Full License 


\section{Abstract}

Introduction: Fulvestrant has demonstrated efficacy in hormone receptor positive (HR+) metastatic breast cancer ( $\mathrm{mBC}$ ), both in first- and second-line settings. In clinical practice, however, fulvestrant has been used as a later-line therapy. The study aimed to assess the efficacy of fulvestrant in women with $\mathrm{mBC}$ in early- versus later-line therapy.

Methods: In this retrospective cohort study, women with HR+ mBC who received fulvestrant between 2003-2019 in Saskatchewan were assessed. A multivariate Cox proportional survival analysis was performed.

Results: 186 women with a median age of 63.5 years were identified; 178 (95.6\%) had hormone resistant $\mathrm{mBC}, 57.5 \%$ had visceral disease, and $43.0 \%$ had received chemotherapy before fulvestrant. 102 (54.8\%) women received $\leq 2$-line-therapy, and $84(45.2 \%)$ received $\geq 3$ line-therapy before fulvestrant. The median time to progression (TTP) was 12 months in the early-treatment vs. 6 months in the later-treatment group, $p=0.015$. Overall survival (OS) from the start of fulvestrant was 26 months in the early-treatment group vs. 16 months in the later-treatment group, $p=0.067$. On multivariate analysis, absence of visceral metastasis, HR: 0.70 (0.50-0.99), was significantly correlated with better TTP, whereas post-fulvestrant chemotherapy, HR: $0.32(0.23-0.47)$, clinical benefit from fulvestrant, HR: $0.44(0.30-0.65)$ and absence of visceral metastasis, HR: 0.70 (0.50-0.97), were correlated with better OS.

Conclusions: Fulvestrant has demonstrated efficacy as both early- and later-line therapy in hormoneresistant $\mathrm{mBC}$. Women with clinical benefit from fulvestrant, who received post-fulvestrant chemotherapy, or had non-visceral disease had better survival.

\section{Introdcution}

Breast cancer is the most common cancer in women worldwide, with about two million women being diagnosed in 2018 (1). Globally, over 600,000 women died from breast cancer in 2018, with breast cancer being responsible for about $15 \%$ of cancer deaths among women (1). Although breast cancer represents a significant global burden, the mortality rates of breast cancer in high-income countries have declined since the 1980 s, largely due to advances in detection and treatment (2).

Breast cancer is divided into three subgroups based on the presence or absence of estrogen receptor (ER), progesterone receptor (PR), or human epidermal growth factor receptor 2 (HER2) overexpression $(3,4)$. Women that have ER/PR positive (HR+) and HER2 negative breast cancer represent over $60 \%$ of newlydiagnosed breast cancer (5). Despite improvements in the outcomes of women with early-stage breast cancer, a subset of women develop metastatic disease. Endocrine therapy is the cornerstone treatment for HR + advanced breast cancer (6). Current endocrine therapies include selective ER modulators (SERMs) that act by blocking the estrogen receptor (such as tamoxifen), non-steroidal and steroidal aromatase inhibitors (NSAls and Als) that reduce estrogen levels by inhibiting the peripheral synthesis of 
estrogen (such as anastrozole, letrozole and exemestane), and the selective ER down-regulator, fulvestrant.

Since endocrine therapy is less toxic than chemotherapy, it is preferable that most women with HR + breast cancer begin treatment with endocrine therapy. Most women with newly-diagnosed advanced breast cancer are preferentially treated with a non-steroidal aromatase inhibitor (letrozole or anastrozole), with or without a CDK 4/6 inhibitor $(7,8,9)$. On progression, exemestane (a steroidal aromatase inhibitor), tamoxifen, or fulvestrant are frequently used as potential second-line agents.

Fulvestrant is a pure ER antagonist, exerting selective ER downregulation, and competitively binding to the $\operatorname{ER}(10,11)$. It is administered as an intramuscular injection (500 mg loading dose on days 1,14 , and 29 of the first month, then maintenance dosing monthly at day $28 \pm 3$ days). The efficacy of first-line fulvestrant in comparison with the aromatase inhibitor anastrozole has been demonstrated in the phase III FALCON trial (12). This trial involved 462 women with metastatic ER positive breast cancer who had not received previous endocrine therapy. At 25.0 months of follow up, women who received fulvestrant had a median progression-free survival of 16.6 months, versus 13.8 months in those that received anastrozole (HR for progression or death: $0.80,95 \% \mathrm{Cl} 0.64-0.99$ ). Fulvestrant and exemestane are equally active and well-tolerated in women with advanced breast cancer who have experienced progression or recurrence on a non-steroidal aromatase inhibitor (13).

Despite a lack of level 1 evidence of efficacy, in clinical practice, fulvestrant has been used in the 3rd and subsequent lines of therapy in women with ER/PR positive metastatic breast cancer. This retrospective, multicenter cohort study using real-world data explores the efficacy of fulvestrant in women with heavilytreated advanced breast cancer. The primary objective of this study was to compare the efficacy of fulvestrant in women who received fulvestrant as an early line of treatment ( $\leq 2$ lines of therapy) to the efficacy in women who received fulvestrant as a later line of treatment ( $\geq 3$ lines of therapy). The secondary objectives were to compare the efficacy of fulvestrant based on: 1) primary versus secondary endocrine resistance, 2) visceral versus non-visceral metastasis, and 3) to determine prognostic factors that correlate with the benefit of fulvestrant and survival.

\section{Methods}

\section{Eligibility Criteria}

This study was approved by the University of Saskatchewan Biomedical Research Ethics Board. Eligible patients were adult women with histologically-documented ER/PR positive breast cancer with metastasis, who had received fulvestrant through a compassionate access program in Saskatchewan provided by AstraZeneca from 2003-2019. Patients were postmenopausal or premenopausal with gonadal suppression. Women who received at least one dose of fulvestrant alone or in combination with a targeted agent were included. In addition, women who had HR+/HER2 + disease and received fulvestrant 
were included. Individual patient medical records were reviewed, and appropriate data was abstracted with a validated abstraction sheet.

\section{Definitions}

Both endocrine therapy and chemotherapy were considered as lines of therapy. Primary endocrine resistance was defined as relapse during the first 2 years of adjuvant endocrine therapy, or progressive disease within the first 6 months of first-line endocrine therapy in metastatic breast cancer. Secondary endocrine resistance was defined as relapse on adjuvant endocrine therapy after 2 years of starting treatment, relapse 12 months after completing adjuvant endocrine therapy, or progressive disease 6 months after starting endocrine therapy in metastatic breast cancer. Patients with visceral metastasis had evidence of disease in any of the visceral organs (lung, liver, brain, or peritoneum). Non-visceral metastasis included metastasis confined to the skin and soft tissue, distant lymph nodes, or bones. Time to progression (TTP) was defined as the time of commencement of fulvestrant until progression of the disease, as defined by the treating oncologist or the last follow up date. Overall survival (OS) was defined as the time from commencement of fulvestrant until death from any cause or the last follow up date. Responses were recorded as per the treating physicians' assessment.

\section{Statistical Analysis}

Categorical data were summarized as frequency and corresponding proportions. For continuous data, frequency, median, inter-quartile range, mean (as appropriate), and standard deviation were calculated. The chi-square test and Student's t-test were performed for the analysis of categorical and continuous variables. Kaplan-Meier and log-rank methods were used to determine TTP and OS. A multivariate Cox proportional hazard model was used to assess prognostic significance of various factors that correlate with TTP and OS. For both TTP and OS, the following variables were examined: previous lines of therapy ( $\leq 2$ vs. $\geq 3$ ), age ( $\leq 50$ vs. $>50$ years), endocrine resistance (primary vs. secondary), comorbid illnesses, World Health Organization (WHO) performance status ( $\geq 2$ vs. $<2$ ), de novo metastatic breast cancer, chemotherapy use prior to fulvestrant, visceral metastasis, combination therapy (targeted agent) and time from diagnosis of advanced breast cancer to start of fulvestrant treatment. In addition, residence (urban vs. rural), secondary cancer, smoking, clinical benefit (partial response plus stable disease vs. progression) and use of chemotherapy after fulvestrant were examined for their correlation with OS. The hazard ratio and its $95 \% \mathrm{Cl}$ were calculated. For the variables examined in the final mathematical model, the proportional hazards assumption was assessed using log-log survival curves. The variables that showed $p<0.20$ on univariate analysis or were considered to be biologically important were fitted into the multivariate model. The threshold of statistical significance was set at $p<0.05$. All patients were followed until August 2020 when data entry was closed. SPSS version 27 was used for statistical analysis (IBM, Armonk, NY).

\section{Results}


Two hundred and fourteen women were identified as being registered in the compassionate access program for fulvestrant in Saskatchewan between March 2003 and January 2019. Twenty-eight women were excluded (three women did not receive any dose of fulvestrant and 25 did not have adequate follow up information). This study included 186 eligible women with advanced HR+ breast cancer, who had received at least one dose of fulvestrant (Figure 1). The median age at the start of fulvestrant treatment was 63.5 years (IQR: 54.0-74.0). Seventeen (9.1\%) women had HR+/HER2+ disease, $81.2 \%$ had a WHO performance status of $0-1$, and $80.6 \%$ of women had a previous diagnosis of early-stage breast cancer, with $89.3 \%$ of those having received adjuvant therapy. One hundred and forty-three $(76.9 \%)$ women had bony metastases, and $57.5 \%$ had visceral metastasis. Among the entire cohort, $43 \%$ of women had received chemotherapy in the metastatic setting before fulvestrant, and $60.2 \%$ of patients received chemotherapy after discontinuing fulvestrant. Overall, $17.7 \%$ of women received fulvestrant with a targeted therapy (mostly a CDK 4/6 inhibitor). One hundred and seventy-eight women had endocrineresistant disease, with $39(21.9 \%)$ having primary resistance and $139(78.1 \%)$ having secondary resistance. Table 1 lists the patient characteristics.

Overall, 102 (54.8\%) women had started fulvestrant after $\leq 2$ lines of therapy in the metastatic setting, and 84 (45.2\%) women received fulvestrant after $\geq 3$ lines of therapy. Significant differences were noted between the two groups with respect to age, pathological diagnosis of metastatic breast cancer, median line of therapy, prior chemotherapy, and median time of diagnosis to start of fulvestrant treatment. The median age of women in early-line therapy was 67 years, versus 60 years in women in the later-line therapy group $(p=0.001)$. The median line of therapy in the group that received fulvestrant after $\leq 2$ lines of therapy was 1 , compared to 4 in the group who received fulvestrant after $\geq 3$ lines of therapy. Women in the $\leq 2$ lines of therapy group started fulvestrant at a median of 24.5 months after diagnosis of advanced breast cancer, compared to 44.0 months in women who received fulvestrant after $\geq 3$ lines of therapy $(p=0.002)$. Women in the early-line group were less likely to have received chemotherapy prior to fulvestrant compared to the later-line group $(17.6 \%$ vs. $73.8 \%, p=0.001)$, and were less likely to have fulvestrant-refractory disease $(20.6 \%$ vs. $38.1 \%, p=0.009)$.

\section{Survival}

Overall, $74.7 \%$ patients discontinued fulvestrant due to disease progression, $10.2 \%$ discontinued due to side effects or patient request and $1.6 \%$ due to other reasons. Women in the early-line group were less likely to have fulvestrant-refractory disease $(20.6 \%$ vs. $38.1 \%, p=0.009)$ compared to the later-line group. The median TTP of the entire cohort was 8 months (95\% Cl: 5.6-10.4). The median TTP was 12 months (9.4-14.6) in women starting fulvestrant after $\leq 2$ lines of therapy, versus 6 months (5.1-6.9) in women with $\geq 3$ lines of therapy $(p=0.015)$ (Figure 2A) [Table 2]. There was no significant difference in median TTP between women who had primary versus secondary endocrine resistance ( 7 vs. 9 months, $p=$ 0.098). Women who received chemotherapy before fulvestrant had a median TTP of 6 months (4.5-7.4), versus 12 months $(8.7-15.3)$ in those who did not receive chemotherapy $(p=0.039)$ (Figure 2B). 
The OS of all women following commencement of fulvestrant was 21 months (16.0-26.0). The OS was 26 months (16.0-36.0) in women starting fulvestrant after $\leq 2$ lines of therapy, versus 16 months (10.5$21.5)$ in women with $\geq 3$ lines of therapy $(p=0.067)$ (Table 2). There was no significant difference in OS based on primary versus secondary endocrine resistance $(p=0.59)$. Patients that had visceral metastasis had a lower OS, 18 months (14.1-21.9), versus 32 months $(23.0-41.0)$ in those without $(p=0.029)$ [Figure 3A]. Patients that received chemotherapy after fulvestrant had a greater OS, 34 months (30.4$37.6)$, versus 8 months $(4.2-11.8)$ in those that did not $(p<0.001)$ (Table 2$)$.

\section{Cox proportional Multivariate analysis}

Table 3 lists factors that correlate with TTP and provides the hazard ratios for univariate and multivariate analysis of TTP. On univariate analysis, lack of prior chemotherapy and early-line treatment significantly correlated with better TTP. However, on multivariate analysis, only the absence of visceral metastasis was significantly correlated with better TTP, HR: 0.70 (95\% Cl: $0.50-0.99)$. Differences in TTP based on the use of chemotherapy prior to fulvestrant or previous lines of therapy when starting fulvestrant were not significant after adjustment for other variables.

Table 4 lists the hazard ratios for univariate and multivariate analysis of OS, after adjustment of important variables. In multivariate analysis, post-fulvestrant chemotherapy, HR: 0.32 (0.23-0.47), clinical benefit from fulvestrant, HR: 0.44 (0.30-0.65), and absence of visceral metastasis, HR: 0.70 (0.50-0.97) were correlated with better OS. The difference in survival based on WHO performance status was not significant in the final multivariate model after adjustment for other variables.

\section{Discussion}

Our results show that fulvestrant is effective in both early-and later-line therapy in advanced HR + breast cancer. Likewise, women with primary and secondary endocrine-resistant disease received benefit from fulvestrant. As anticipated, the benefit was more pronounced in women who received fulvestrant as an early-line treatment compared to those who received it as a later-line of treatment. Our real-world study cohort treated with fulvestrant had a TTP of 8 months and an overall survival of 21 months. This is comparable to the outcomes of patients in the (CONFIRM) trial, in which participants who were treated with second-line $500 \mathrm{mg}$ of fulvestrant had a progression-free survival of 6.5 months and an overall survival of 26.4 months $(14,15)$.

In our study, the group of patients who received fulvestrant as an early-line therapy had a significantly better TTP of 12 months, compared to 6 months in those who received fulvestrant as a later-line therapy; however, on multivariate analysis, this difference was not significant after adjustment of other prognostic variables. Other studies have shown a positive correlation between previous lines of therapy and survival $(16,17)$. For example, a Japanese study showed $20 \%$ relative improvement in time to treatment failure associated with line of therapy; however, the numerical differences based on treatment line were small, 5.8 months in first and second line fulvestrant treatment and 4.6 months beyond the fourth line (16). Likewise, our results showed that women who received fulvestrant as an earlier line of therapy had a 
trend of better overall survival compared to those who received fulvestrant as a later line of therapy (26 vs. 16 months), with a $26 \%$ reduction in mortality. This trend towards better survival in women who received fulvestrant as an early-line therapy may be because the patients starting fulvestrant at a later line of therapy were heavily pre-treated in comparison, and had a longer standing disease that may not respond as well to subsequent therapy. Patients taking fulvestrant after $\leq 2$ lines of therapy had started fulvestrant after a median duration of 24.5 months after being diagnosed with metastatic breast cancer, and had a median overall survival of 26 months after starting fulvestrant. In comparison, patients taking fulvestrant after $\geq 3$ lines of therapy had been diagnosed with metastatic breast cancer for a median duration of 44 months before starting fulvestrant, with a median overall survival of 16 months. Interestingly, patients starting fulvestrant at a later line of therapy had a better overall survival from the time of the diagnosis of advanced breast cancer (median OS of 73 months vs. 48 months). However, this difference in overall survival from the time of diagnosis may be because the group of patients taking fulvestrant as a later line of therapy were younger and had a high rate of chemotherapy prior to commencement of fulvestrant.

Similar to the FALCON trial, fulvestrant was found to be more effective in patients with non-visceral metastases (12). Our results show that TTP and OS were shorter in patients with visceral metastases compared to those with bone and soft tissue metastases. Other real-world studies have also shown decreased disease control with fulvestrant in patients with visceral metastases $(17,18,19)$. It is important to note that patients with primary versus secondary endocrine-resistant disease did not have significant differences in TTP or OS. Other studies have demonstrated a decreased response to fulvestrant based on previous endocrine insensitivity $(17,20)$. This study also shows that patients taking chemotherapy after discontinuing fulvestrant had a significantly longer median overall survival of 34 months, compared to 8 months in patients who did not receive chemotherapy, showing a $68 \%$ relative reduction in mortality. In addition, independent of the use of chemotherapy following progression on fulvestrant, clinical benefit from fulvestrant (defined as partial response or stable disease) was strongly correlated with better overall survival, with an approximately $56 \%$ relative reduction in mortality compared to those who progressed on fulvestrant.

Despite the considerable success of fulvestrant, one limitation is that it must be administered by intramuscular injection. Therefore, there is a need to improve delivery of fulvestrant by developing orally bioavailable selective estrogen receptor degraders (SERDs). Current oral SERDs in phase III development include Elacestrant (ClinicalTrials.gov Identifier: NCT03778931) and GDC-9545 (NCT04546009). Although our study participants were primarily using fulvestrant monotherapy, based on promising new data on CDK 4/6 inhibitors, it is recommended that CDK 4/6 inhibitors be added in combination in patients who have not previously received them. The benefit of CDK 4/6 inhibitors to fulvestrant treatment has been well established by significant increases in both PFS and OS (6).

Our study provides information on the efficacy of fulvestrant in real-world clinical practice; however, it is important to highlight some limitations. First, it was a retrospective study, which carries some limitations. The study population was treated over several years, and only about $20 \%$ of patients received 
combination therapy, so this may not adequately reflect the recent practice of combination treatment, such as the addition of CDK 4/6 inhibitors to the standard of therapy. Another limitation is that the endpoints were based on the treating physician's determined assessment, which can be difficult to standardize. This study was not able to investigate the effectiveness of fulvestrant in comparison to other treatment arms. However, one of the major strengths of this study is that it had a generalized inclusion criteria, and all of the women who received fulvestrant and had adequate follow up in Saskatchewan were assessed, reflecting a population-based study. The results of this study further validate the effectiveness of fulvestrant in previously-treated $\mathrm{HR}+$ breast cancer, and provide useful information of the efficacy of fulvestrant based on patient characteristics.

\section{Conclusions}

Fulvestrant has demonstrated efficacy as both an early and later-line therapy in metastatic breast cancer. The OS in both early and later lines of therapy is similar, but women who received $\leq 2$ lines therapy prior to fulvestrant had a better TTP after starting fulvestrant. Women with visceral disease at the commencement of fulvestrant, regardless of previous lines of treatment, had a shorter duration of disease control and OS. In addition, clinical benefit from fulvestrant and the use of chemotherapy following fulvestrant were correlated with better OS. This study showed no difference in TTP or OS based on endocrine sensitivity.

\section{Declarations}

\section{Acknowledgements}

We are appreciative of the Mach-Gaensslen Foundation of Canada and the University of Saskatchewan College of Medicine for supporting this research. We are also thankful to the Saskatchewan Cancer Agency for access to the data.

\section{Disclosure}

The authors declare no conflicts of interest.

\section{References}

1. Ferlay J, Colombet M, Soerjomataram I, Mathers C, Parkin DM, Piñeros M, et al. Estimating the global cancer incidence and mortality in 2018: GLOBOCAN sources and methods. Int J Cancer. 2019 Apr 15;144(8):1941-53.

2. Brenner DR, Weir HK, Demers AA, Ellison LF, Louzado C, Shaw A, et al. Projected estimates of cancer in Canada in 2020. CMAJ. 2020 Mar 2;192(9):E199-205.

3. Parise CA, Bauer KR, Brown MM, Caggiano V. Breast cancer subtypes as defined by the estrogen receptor (ER), progesterone receptor (PR), and the human epidermal growth factor receptor 2 (HER2) 
among women with invasive breast cancer in California, 1999-2004. Breast J 2009; 15:593.

4. Hammond ME, Hayes DF, Dowsett M, et al. American Society of Clinical Oncology/College of American Pathologists guideline recommendations for immunohistochemical testing of estrogen and progesterone receptors in breast cancer. J Clin Oncol 2010; 28:2784.

5. Female Breast Cancer Subtypes - Cancer Stat Facts [Internet]. SEER. [cited 2020 Dec 18]. Available from: https://seer.cancer.gov/statfacts/html/breast-subtypes.html

6. Cardoso F, Costa A, Senkus E, et al. 3rd ESO-ESMO International Consensus Guidelines for Advanced Breast Cancer (ABC 3). Ann Oncol. 2017 Jan 1;28(1):16-33.

7. Finn RS, Martin M, Rugo HS, et al. Palbociclib and Letrozole in Advanced Breast Cancer. N Engl J Med 2016; 375:1925.

8. Goetz MP, Toi M, Campone M, et al. MONARCH 3: Abemaciclib As Initial Therapy for Advanced Breast Cancer. J Clin Oncol 2017; 35:3638.

9. Hortobagyi GN, Stemmer SM, Burris HA, et al. Updated results from MONALEESA-2, a phase III trial of first-line ribociclib plus letrozole versus placebo plus letrozole in hormone receptor-positive, HER2negative advanced breast cancer. Ann Oncol 2018; 29:1541.

10. Ciruelos E, Pascual T, Arroyo Vozmediano ML, et al. The therapeutic role of fulvestrant in the management of patients with hormone receptor positive breast cancer. Breast 2014; 23: 201-208.

11. Rugo HS, Rumble RB, Macrae E, et al. Endocrine therapy for hormone receptor-positive metastatic breast cancer: American Society of Clinical Oncology Guideline. J Clin Oncol 2016;34: 3069-3103.

12. Robertson JF, Bondarenko IM, Trishkina E, et al. Fulvestrant $500 \mathrm{mg}$ versus anastrozole $1 \mathrm{mg}$ for hormone receptor-positive advanced breast cancer (FALCON): an international, randomised, doubleblind, phase 3 trial. Lancet 2016; 388:2997.

13. Chia S, Gradishar W, Mauriac L, et al. Double-blind, randomized placebo controlled trial of fulvestrant compared with exemestane after prior nonsteroidal aromatase inhibitor therapy in postmenopausal women with hormone receptor-positive, advanced breast cancer: results from EFECT. J Clin Oncol 2008; 26:1664.

14. Di Leo A, Jerusalem G, Petruzelka L, Torres R, Bondarenko IN, Khasanov R, et al. Final overall survival: fulvestrant 500 mg vs 250 mg in the randomized CONFIRM trial. J Natl Cancer Inst. 2014 Jan;106(1):djt337.

15. Di Leo A, Jerusalem G, Petruzelka L, Torres R, Bondarenko IN, Khasanov R, et al. Results of the CONFIRM phase III trial comparing fulvestrant $250 \mathrm{mg}$ with fulvestrant $500 \mathrm{mg}$ in postmenopausal women with estrogen receptor-positive advanced breast cancer. J Clin Oncol. 2010 Oct 20;28(30):4594-600.

16. Kawaguchi H, Masuda N, Nakayama T, Aogi K, Anan K, Ito Y, et al. Outcomes of fulvestrant therapy among japanese women with advanced breast cancer: a retrospective multicenter cohort study (JBCRG-C06; Safari). Breast Cancer Res Treat. 2017 Jun;163(3):545-54.

17. Moscetti L, Fabbri MA, Natoli C, Vici P, Gamucci T, Sperduti I, et al. Fulvestrant 500 milligrams as endocrine therapy for endocrine sensitive advanced breast cancer patients in the real world: the 
Ful500 prospective observational trial. Oncotarget. 2017 Apr 20;8(33):54528-36.

18. Ozawa H, Sata A, Fukui R, Bun A, Higuchi T, Fujimoto Y, et al. A Single-centre, Retrospective, Observational Analysis of Fulvestrant for Recurrent/metastatic Breast Cancer According to Metastatic Site. Anticancer Res. 2019 Oct;39(10):5653-62.

19. Lei W, Li H, Song G, Zhang R, Ran R, Yan Y, et al. Efficacy and Safety of Fulvestrant $500 \mathrm{mg}$ in Hormone-receptor Positive Human Epidermal Receptor 2 Negative Advanced Breast Cancer: A Realworld Study in China. J Cancer. 2020 Sep 23;11(22):6612-22.

20. Skinner KE, Olufade T, Walker MS, Schwartzberg LS. Real-world effectiveness of fulvestrant monotherapy as first endocrine treatment in patients with metastatic breast cancer. Breast J. 2020 Feb;26(2):112-9.

\section{Tables}

Table 1: Patient characteristics and treatment response to fulvestrant. 
Variables $n=186$

(\%)

Study Cohort $\leq 2$ lines of therapy

$\mathrm{n}=102$

(54.8\%)

(\%) $\geq 3$ lines of

therapy

$n=84(45.2 \%)$

(\%)

Demographics

Median age

63.5 (IQR:

54.0-74.0)

$>50$ years

157 (84.4)

93 (91.2)

64 (76.2)

0.007

Rural residence

94 (50.5)

$53(52)$

$41(48.8)$

0.76

Comorbid illness

94 (50.5)

$58(57)$

$36(42.8)$

0.24

Secondary cancer

$22(11.8)$

14 (13.7)

$8(9.5)$

0.49

WHO performance status 0-1

151 (81.2)

$82(80.3)$

$69(82.1)$

0.85

Smoking History

69 (37.1)

$44(43.1)$

$25(29.8)$

0.21

History of early-stage breast cancer

$150(80.6)$

85 (83.3)

$65(77.4)$

0.35

Bilateral breast cancer

$12(8.0)$

$10(11.7)$

$2(3.0)$

0.12

Surgery for early-stage breast cancer

$\begin{array}{lllll}\text { Mastectomy } & 94(62.6) & 53(62.3) & 41(63.0) & 0.37 \\ \text { Lumpectomy } & 36(24.0) & 19(22.3) & 17(26.1) & 0.70 \\ \text { Bilateral mastectomy } & 20(13.3) & 13(15.3) & 7(10.7) & 0.33\end{array}$

Stage of early-breast cancer

\begin{tabular}{|lllll|}
\hline I & $35(23.3)$ & $18(21.1)$ & $17(26.1)$ & 0.55 \\
\hline III & $71(47.3)$ & $40(47.0)$ & $31(47.8)$ & 1.0 \\
Received Adjuvant therapy & $44(29.3)$ & $27(31.7)$ & $17(26.1)$ & 0.47 \\
\hline Adjuvant chemotherapy & $134(89.3)$ & $75(88.2)$ & $59(90.7)$ & 0.79 \\
\hline Adjuvant endocrine therapy & $81(54.0)$ & $44(51.7)$ & $37(56.9)$ & 0.62 \\
\hline Adjuvant radiation therapy & $110(73.3)$ & $67(78.8)$ & $43(66.2)$ & 0.09 \\
\hline Diagnosis of metastatic disease & $96(64.0)$ & $53(62.4)$ & $43(66.2)$ & 0.73 \\
Clinical & & & & \\
\hline Pathological & $39(21.0)$ & $30(29.4)$ & $9(10.7)$ & 0.002 \\
\hline
\end{tabular}




\begin{tabular}{|c|c|c|c|c|}
\hline \multicolumn{5}{|l|}{ Receptor status } \\
\hline Estrogen receptor positive & $183(98.4)$ & $100(98.0)$ & $83(98.8)$ & 1.0 \\
\hline Progesterone receptor positive & $152(81.7)$ & $84(82.4)$ & $68(81.0)$ & 0.85 \\
\hline HER2 overexpression & $17(9.1)$ & $7(6.9)$ & $10(12.3)$ & 0.30 \\
\hline Visceral metastases & $107(57.5)$ & $53(52.0)$ & $54(64.3)$ & 0.10 \\
\hline \multicolumn{5}{|l|}{ Location of Metastases } \\
\hline Bone & $143(76.9)$ & 75 (73.5) & $68(81.0)$ & 0.29 \\
\hline Lung & $78(40.9)$ & $39(38.2)$ & $39(46.4)$ & 0.29 \\
\hline Liver & $58(31.2)$ & $27(26.5)$ & $31(36.9)$ & 0.15 \\
\hline Skin or soft tissue & $47(25.3)$ & $22(21.6)$ & $25(29.8)$ & 0.23 \\
\hline Nodal & $27(14.5)$ & $10(9.8)$ & $17(20.2)$ & 0.06 \\
\hline Brain & $7(3.8)$ & $2(2.0)$ & $5(6.0)$ & 0.24 \\
\hline Peritoneal & $5(2.7)$ & $3(2.9)$ & $2(2.4)$ & 1.0 \\
\hline Median line of therapy & 2 (range 0-8) & $1(0-2)$ & $4(3-8)$ & $<0.001$ \\
\hline Four or more lines of therapy & $42(22.5)$ & 0 & $56(71.8)$ & $<0.001$ \\
\hline $\begin{array}{l}\text { Median time from diagnosis to start } \\
\text { of fulvestrant }\end{array}$ & $\begin{array}{l}34 \text { (IQR: } 20.0- \\
63.0)\end{array}$ & $\begin{array}{l}24.5(12.0- \\
44.5)\end{array}$ & $44(28.0-87.0)$ & 0.002 \\
\hline $\begin{array}{l}\text { Received chemotherapy prior to } \\
\text { fulvestrant }\end{array}$ & $80(43.0)$ & $18(17.6)$ & $62(73.8)$ & 0.001 \\
\hline Combination treatment & $34(18.3)$ & $21(20.6)$ & $13(15.5)$ & 0.44 \\
\hline Targeted Therapy & $33(17.7)$ & $21(100)$ & $12(92)$ & 0.32 \\
\hline Endocrine Resistant & $178(95.7)$ & $95(93.1)$ & $83(98.8)$ & 0.07 \\
\hline Primary & $39(21.9)$ & $27(28.4)$ & $12(14.4)$ & 0.04 \\
\hline \multicolumn{5}{|l|}{$\begin{array}{l}\text { Reason for discontinuation of } \\
\text { fulvestrant }\end{array}$} \\
\hline Progression & $139(74.7)$ & $71(69.6)$ & $68(81.0)$ & 0.09 \\
\hline Side effects or patient request & $19(10.2)$ & $13(12.7)$ & $6(7.2)$ & 0.23 \\
\hline Others & $3(1.6)$ & $2(1.9)$ & $1(1.3)$ & 1.0 \\
\hline \multicolumn{5}{|l|}{ Best Response to fulvestrant } \\
\hline Complete response & $2(1.1)$ & $2(1.9)$ & 0 & 0.50 \\
\hline Partial response & $24(12.9)$ & $14(13.8)$ & $10(11.9)$ & 0.82 \\
\hline
\end{tabular}




\begin{tabular}{|lllll|}
\hline Stable disease & $106(57)$ & $65(63.7)$ & $41(48.8)$ & 0.05 \\
\hline Progressive disease & $53(28.6)$ & $21(20.6)$ & $32(38.1)$ & 0.009 \\
\hline Unknown & $1(0.5)$ & 0 & $1(1.2)$ & 0.45 \\
\hline $\begin{array}{l}\text { Received chemotherapy post } \\
\text { fulvestrant }\end{array}$ & $112(60.2)$ & $57(55.9)$ & $55(65.5)$ & 0.22 \\
\hline
\end{tabular}

Table 2: Time to progression and overall survival of patients treated with fulvestrant.

\begin{tabular}{|lllll|}
\hline & TTP & & OS & \\
\hline Variables & Months $(95 \% \mathrm{Cl})$ & P value & Months $(95 \% \mathrm{Cl})$ & P value \\
\hline Primary resistance & $7(2.8-11.2)$ & 0.098 & $32(17.2-46.8)$ & 0.592 \\
Secondary resistance & $9(6.1-11.9)$ & & $21(15.6-26.4)$ & \\
Chemotherapy before fulvestrant & $6(4.5-7.5)$ & 0.039 & $21(10.7-31.3)$ & 0.519 \\
No chemotherapy before fulvestrant & $12(8.7-15.3)$ & & $21(14.8-27.2)$ & \\
Chemotherapy after fulvestrant & - & - & $34(30.4-37.6)$ & $<0.001$ \\
No chemotherapy after fulvestrant & - & & $8(4.2-11.8)$ & \\
50 or younger & $10(4.5-15.5)$ & 0.829 & $32(23.3-40.7)$ & 0.500 \\
51 or older & $8(5.9-10.1)$ & & $21(16.3-25.7)$ & \\
Visceral Metastasis & $7(5.1-8.9)$ & 0.142 & $18(14.1-21.9)$ & 0.029 \\
No Visceral Metastasis & $11(7.9-14.1)$ & & $32(23.0-41.0)$ & \\
$\leq 2$ previous therapies & $12(9.4-14.6)$ & 0.015 & $26(16.0-36.0)$ & 0.067 \\
$\geq 3$ previous therapies & $6(5.1-6.9)$ & & $16(10.5-21.5)$ & \\
Overall & $8(5.6-10.4)$ & & $21(16.0-26.0)$ & \\
& & & & \\
\hline
\end{tabular}

Table 3: Cox proportional univariate and multivariate analysis of variables correlate with time to progression. 


\begin{tabular}{|c|c|c|c|c|}
\hline & Univariate & & Multivariate & \\
\hline Variables & $\mathrm{HR}(95 \% \mathrm{Cl})$ & $\begin{array}{l}P \\
\text { value }\end{array}$ & $\mathrm{HR}(95 \% \mathrm{Cl})$ & $\begin{array}{l}\mathrm{P} \\
\text { value }\end{array}$ \\
\hline Age $<51$ & $\begin{array}{l}0.952(0.597- \\
1.517)\end{array}$ & 0.835 & - & - \\
\hline WHO PS $<2$ & $0.89(0.53-1.49)$ & 0.680 & - & - \\
\hline Comorbid IIIness & $0.78(0.55-1.10)$ & 0.170 & - & - \\
\hline Early-Stage Breast cancer & $\begin{array}{l}0.790(0.524- \\
1.191)\end{array}$ & 0.260 & - & - \\
\hline $\begin{array}{l}\text { Secondary Endocrine } \\
\text { Resistance }\end{array}$ & $0.728(0.493-1.077)$ & 0.112 & $\begin{array}{l}0.717(0.480- \\
1.071)\end{array}$ & 0.104 \\
\hline $\begin{array}{l}\text { Chemotherapy prior to } \\
\text { Fulvestrant }\end{array}$ & $\begin{array}{l}0.710(0.506- \\
0.996)\end{array}$ & 0.047 & $\begin{array}{l}0.802(0.540- \\
1.191)\end{array}$ & 0.274 \\
\hline Non-Visceral Metastasis & $\begin{array}{l}0.783(0.557- \\
1.099)\end{array}$ & 0.158 & $\begin{array}{l}0.701(0.495- \\
0.994)\end{array}$ & 0.046 \\
\hline$\leq 2$ previous therapies & $\begin{array}{l}0.672(0.480- \\
0.940)\end{array}$ & 0.020 & $\begin{array}{l}0.775(0.517- \\
1.161)\end{array}$ & 0.216 \\
\hline Concurrent RX & $\begin{array}{l}0.714(0.462- \\
1.104)\end{array}$ & 0.130 & $\begin{array}{l}0.745(0.468- \\
1.187)\end{array}$ & 0.216 \\
\hline Time to Fulvestrant & $\begin{array}{l}0.732(0.523- \\
1.024)\end{array}$ & 0.068 & - & - \\
\hline
\end{tabular}

Table 4: Cox proportional univariate and multivariate analysis of variables correlate with overall survival. 


\begin{tabular}{|c|c|c|c|c|}
\hline & Univariate & & Multivariate & \\
\hline Variables & $\mathrm{HR}(95 \% \mathrm{Cl})$ & $\begin{array}{l}P \\
\text { value }\end{array}$ & $\mathrm{HR}(95 \% \mathrm{Cl})$ & $P$ value \\
\hline Rural City & $0.81(0.59-1.15)$ & 0.197 & $\begin{array}{l}0.80(0.58- \\
1.12)\end{array}$ & 0.20 \\
\hline Age & $\begin{array}{l}0.857(0.542- \\
1.353)\end{array}$ & 0.507 & - & - \\
\hline WHO PS $<2$ & $\begin{array}{l}0.538(0.358- \\
0.806)\end{array}$ & 0.003 & $\begin{array}{l}0.73(0.48- \\
1.11)\end{array}$ & 0.15 \\
\hline Comorbid IIIness & $\begin{array}{l}0.869(0.633- \\
1.193)\end{array}$ & 0.387 & - & - \\
\hline Secondary Cancer & $0.70(0.42-1.16)$ & 0.172 & $\begin{array}{l}0.65(0.38- \\
1.10)\end{array}$ & 0.11 \\
\hline No Smoking & $0.86(0.62-1.21)$ & 0.402 & - & - \\
\hline Early-Stage Breast cancer & $\begin{array}{l}0.940(0.630- \\
1.402)\end{array}$ & 0.760 & - & - \\
\hline Primary Endocrine Resistance & $0.90(0.60-1.33)$ & 0.597 & - & - \\
\hline $\begin{array}{l}\text { Chemotherapy prior to } \\
\text { Fulvestrant }\end{array}$ & $\begin{array}{l}0.901(0.653- \\
1.243)\end{array}$ & 0.525 & - & - \\
\hline Chemotherapy post fulvestrant & $\begin{array}{l}0.425(0.305- \\
0.593)\end{array}$ & $<0.001$ & $\begin{array}{l}0.32(0.23- \\
0.47)\end{array}$ & $<0.0001$ \\
\hline Non-Visceral Metastasis & $\begin{array}{l}0.701(0.506- \\
0.970)\end{array}$ & 0.032 & $0.70(0.50-0.97)$ & 0.03 \\
\hline$\leq 2$ previous therapies & $\begin{array}{l}0.747(0.544- \\
1.026)\end{array}$ & 0.072 & $\begin{array}{l}0.76(0.55- \\
1.05)\end{array}$ & 0.10 \\
\hline Clinical Benefit & $\begin{array}{l}0.574(0.402- \\
0.819)\end{array}$ & 0.002 & $\begin{array}{l}0.44(0.30- \\
0.65)\end{array}$ & $<0.0001$ \\
\hline
\end{tabular}

\section{Figures}




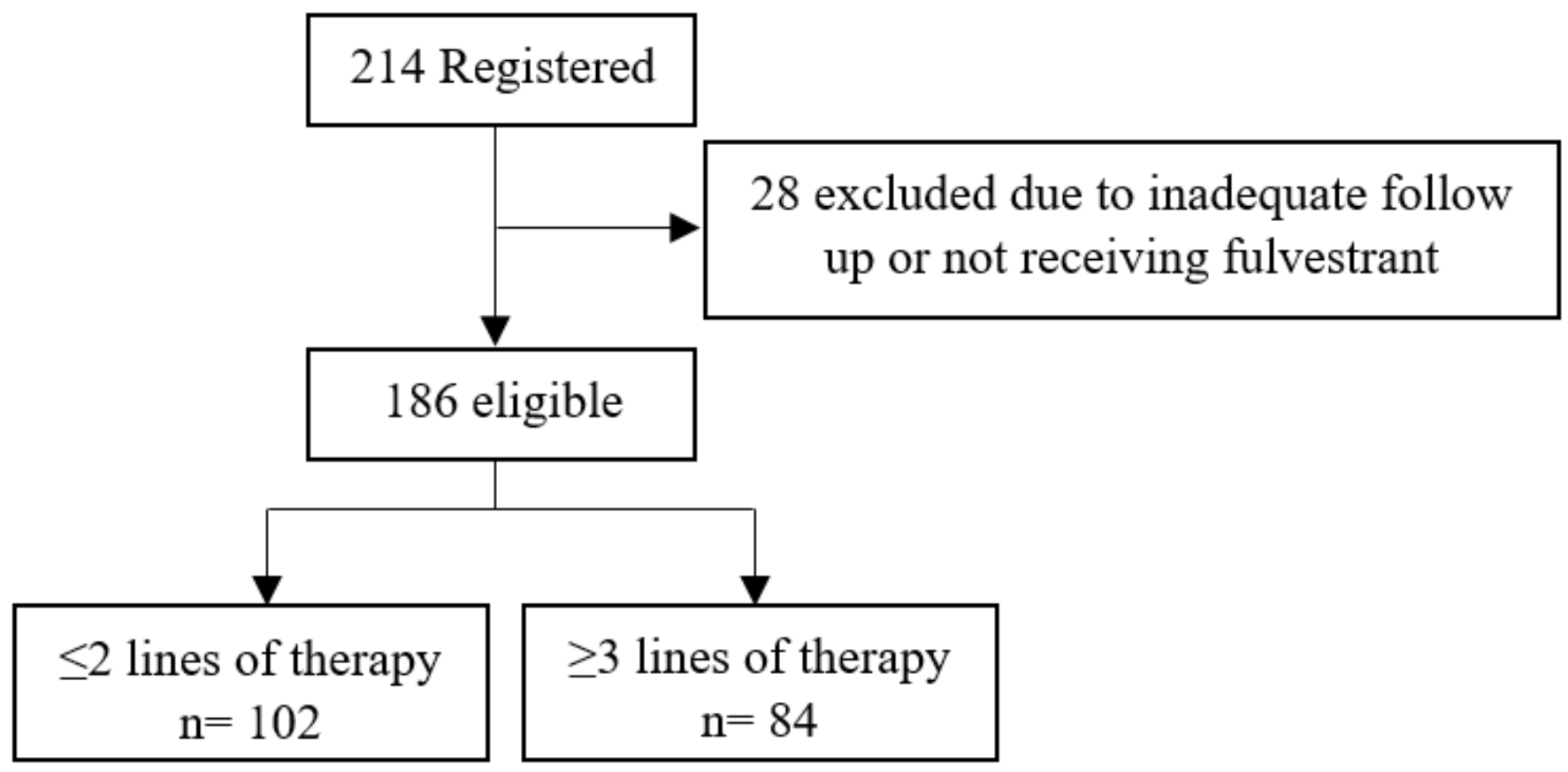

Figure 1

Flow chart of study participants who received fulvestrant 


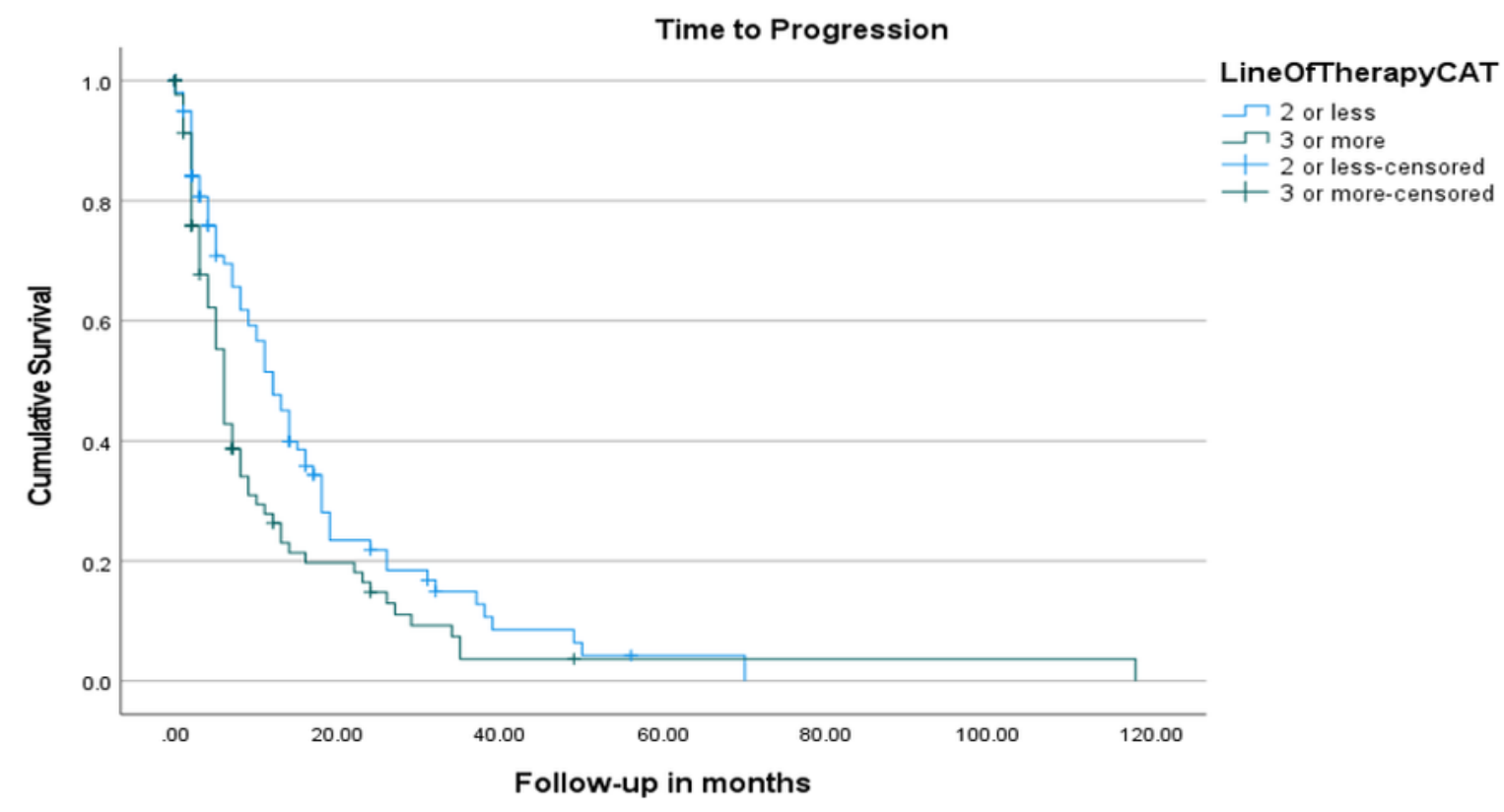

B

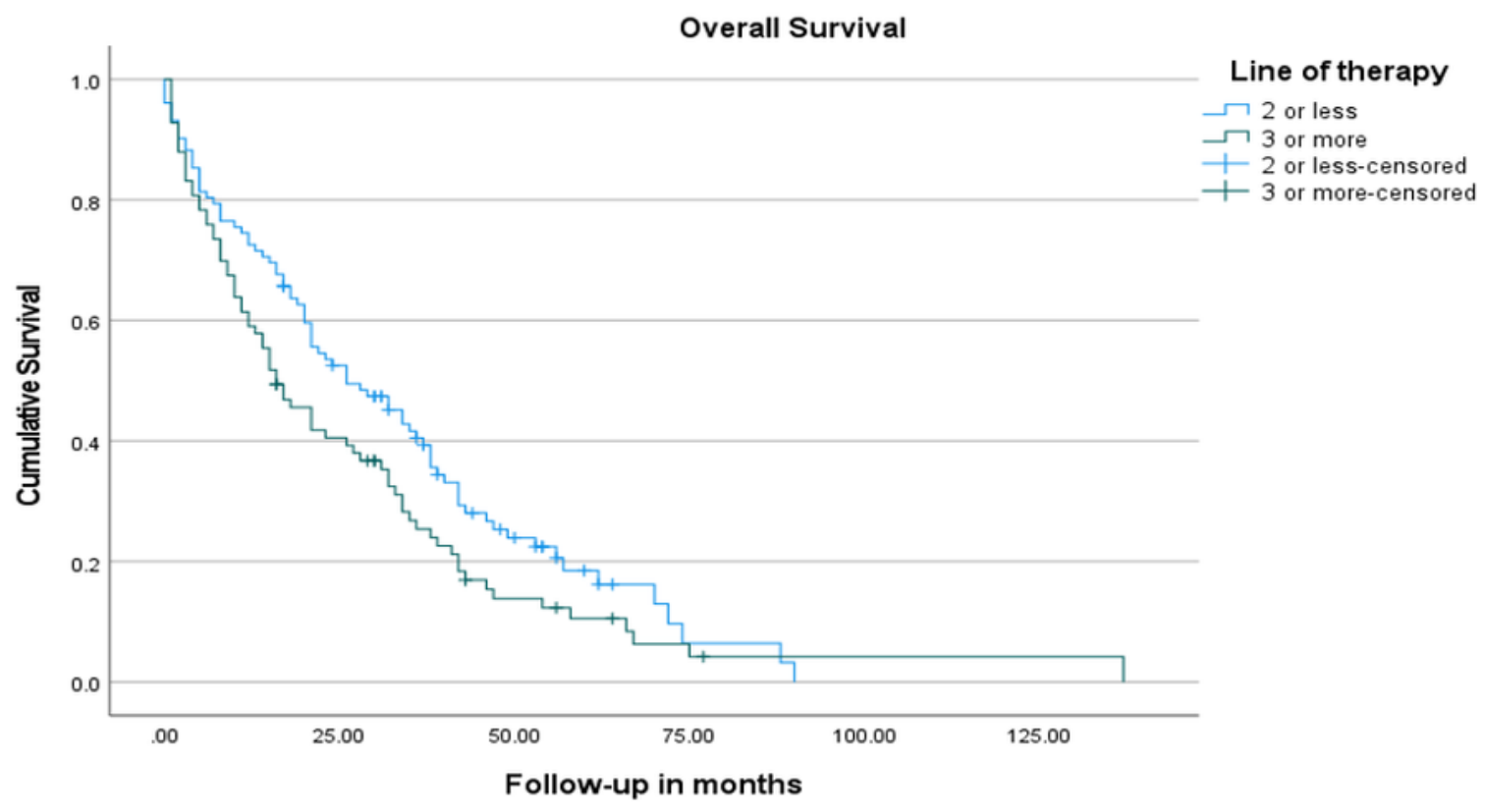

Figure 2

Kaplan-Meier survival curves for time to progression (A) and overall survival (B) stratified by line of therapy. 
A

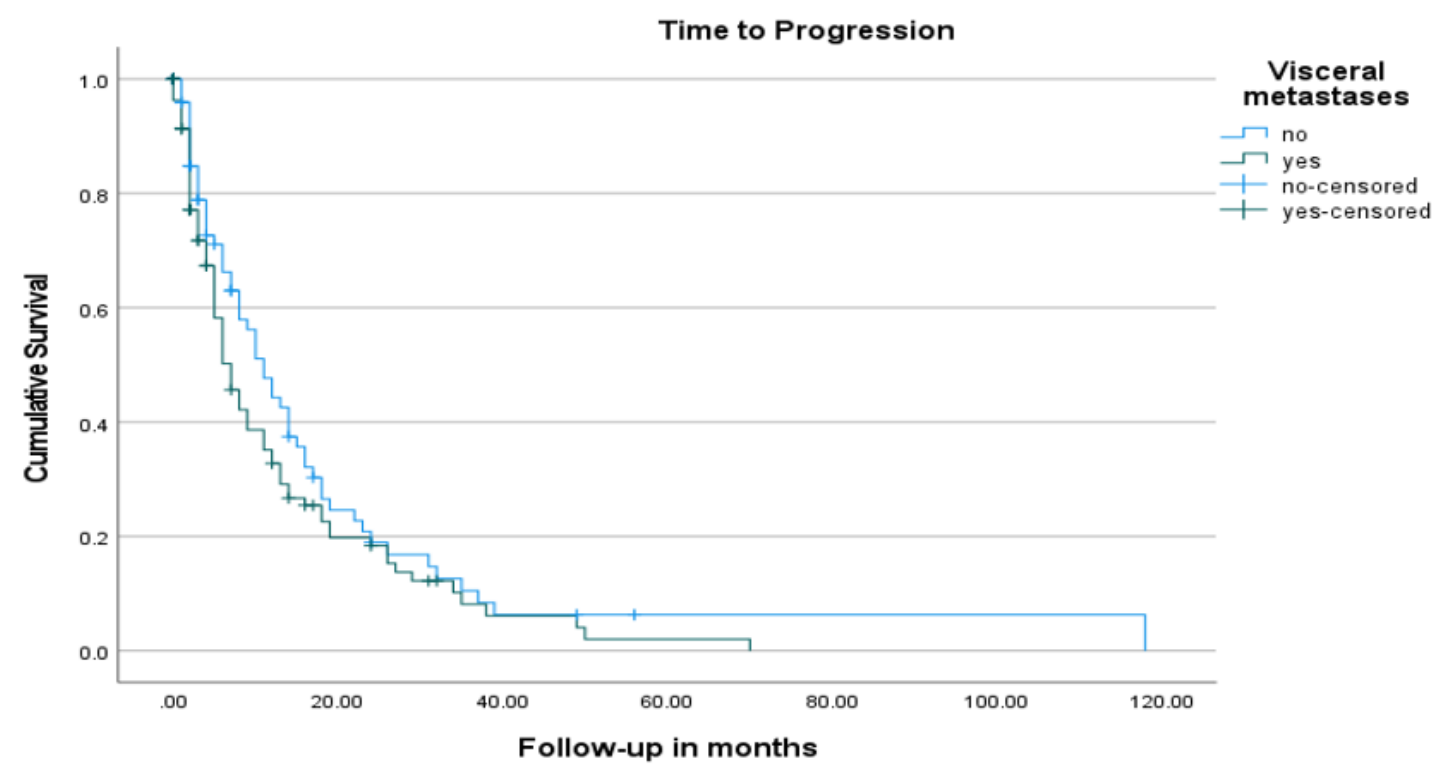

B

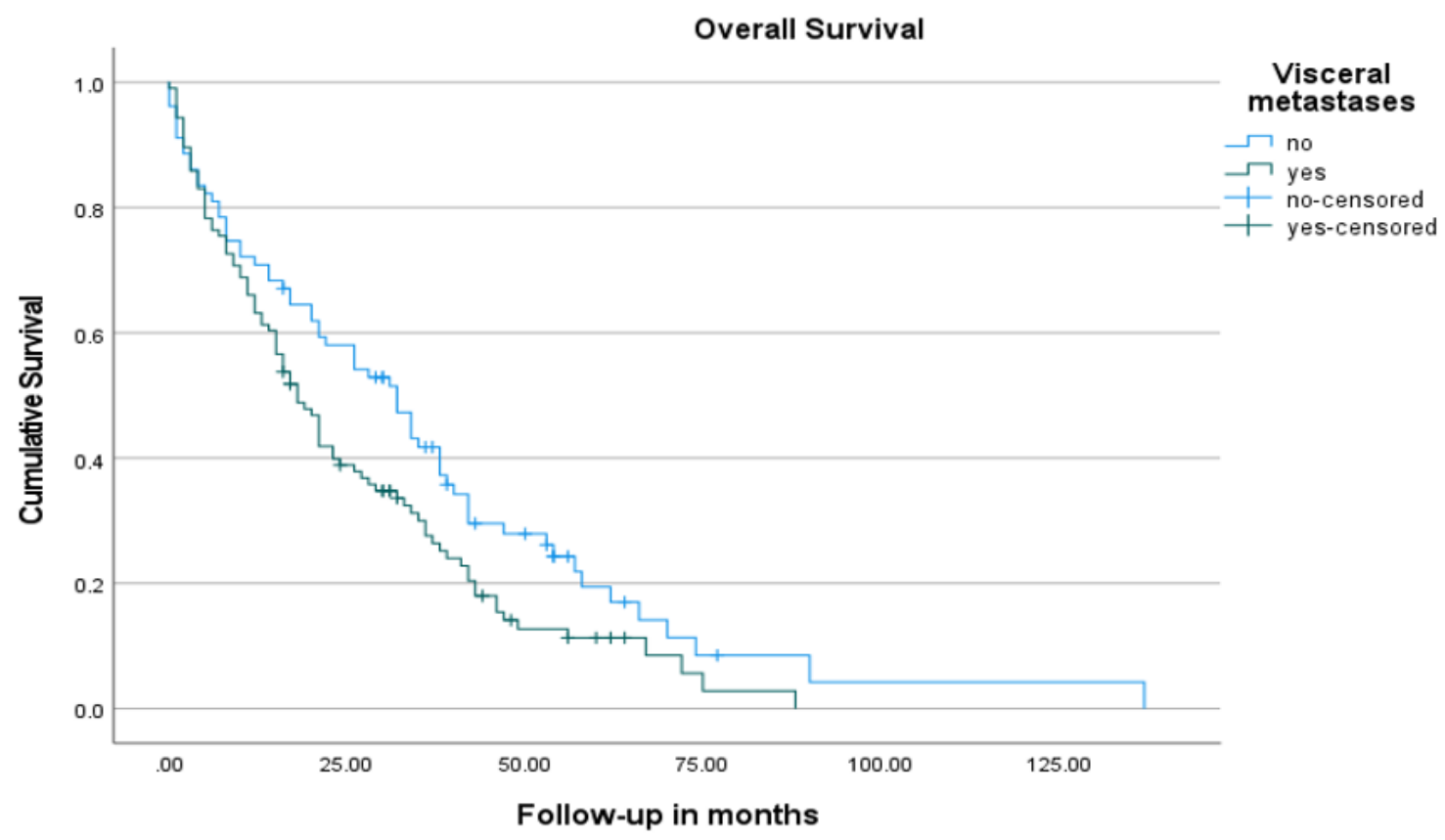

Figure 3

Kaplan-Meier survival curves for time to progression (A) and overall survival (B) stratified by the presence of visceral metastasis. 